\title{
Refining near-native protein-protein docking decoys by local resampling and energy minimization
}

\author{
Shide Liang ${ }^{1,2,3}$, Guangce Wang ${ }^{3}$, and Yaoqi Zhou ${ }^{1,2, *}$ \\ ${ }^{1}$ Indiana University School of Informatics, Indiana University-Purdue University, Indianapolis, \\ Indiana 46202 \\ ${ }^{2}$ Center for Computational Biology and Bioinformatics, Indiana University School of Medicine, \\ Indianapolis, Indiana 46202 \\ ${ }^{3}$ Key Laboratory of Experimental Marine Biology, Institute of Oceanology, Chinese Academy of \\ Sciences, Qingdao 266071, People's Republic of China
}

\begin{abstract}
How to refine a near-native structure to make it closer to its native conformation is an unsolved problem in protein-structure and protein-protein complex-structure prediction. In this article, we first test several scoring functions for selecting locally resampled near-native protein-protein docking conformations and then propose a computationally efficient protocol for structure refinement via local resampling and energy minimization. The proposed method employs a statistical energy function based on a Distance-scaled Ideal-gas REference state (DFIRE) as an initial filter and an empirical energy function EMPIRE (EMpirical Protein-InteRaction Energy) for optimization and re-ranking. Significant improvement of final top-1 ranked structures over initial near-native structures is observed in the ZDOCK 2.3 decoy set for Benchmark 1.0 (74\% whose global rmsd reduced by $0.5 \AA$ or more and only $7 \%$ increased by $0.5 \AA$ or more). Less significant improvement is observed for Benchmark 2.0 (38\% versus 33\%). Possible reasons are discussed.
\end{abstract}

\section{Keywords}

docking structure refinement; local resampling; energy score

\section{INTRODUCTION}

One problem facing both protein-structure prediction and protein docking is how to reduce the root-mean-squared distance (rmsd) of near-native structure of a protein or proteinprotein complex from the corresponding native (complex) structure. In fact, structural refinement is the major bottleneck for high-resolution structure prediction ${ }^{1,2}$ even though some progresses are made. ${ }^{3-11}$ For complex-structure prediction by docking, refinement is necessary because docked decoys often contain severe atomic clashes to increase the chance of sampling near-native structures in the initial stage of global conformational search.

Refinement for docking typically focuses on side-chain optimization, removal of atomic clashes, and optimization of electrostatic energy. ${ }^{12-16}$ For example, RDOCK, ${ }^{17}$ a refinement 
protocol for docking decoys generated by the docking program ZDOCK, ${ }^{18}$ minimizes van der Waals, polar, and charged interactions in three separate stages. RosettaDock ${ }^{19-21}$ is a program that performs protein-protein docking with simultaneous optimization of rigid-body displacement and side-chain conformations by Monte Carlo simulations. ${ }^{19}$ It contains an empirical energy function made of physical and knowledge-based energy terms and optimized for protein-protein binding and docking studies. ${ }^{22,23}$ The method focuses on sidechain optimization in the second-stage all-atom refinement. 3D-DOCK ${ }^{24}$ (formerly FTDOCK that uses Fourier transform to speed up rigid body docking), uses MultiDock ${ }^{25}$ (Multiple copy side-chain refinement of Protein-Protein interfaces) to refine structures through detailed empirical energetics and rotamer-based side-chain positions.

However, refinement by energy minimization often leads to an unrealistic bias toward docked structures with large interfaces. This makes it difficult to identify the near-native complexes with a small interface from non-native decoys with large interfaces. ${ }^{26}$ Recently, we found that the unrealistic dependence of binding affinity of docking decoys on the buried solvent accessible surface area of interface can be removed by using a simple reference state. The resulting energy function, called EMPIRE ${ }^{27}$ (EMpirical Protein-InteRaction Energy), provides a significantly improved success rate in near-native selections when applied to RosettaDock and refined ZDOCK docking decoys. These docking decoys are protein complex structures generated by the docking programs RosettaDock ${ }^{19}$ and ZDOCK, ${ }^{18}$ respectively.

Most studies in docking refinement limited to side-chain and energy minimization. Camacho and $\mathrm{Vajda}^{28}$ developed a multistage refinement protocol by local resampling with a van der Waals constraint and staged energy minimization (the desolvation and electrostatic energy first and followed by van der Waals energy). They have addressed a question that often has been overlooked: Can resampling and refinement around a given near-native structure improve docking prediction?

In this article, we take several more recently developed energy functions to test their abilities for refining near-native structures. In addition to EMPIRE, ${ }^{27}$ Rosetta-Dock, ${ }^{19}$ and RDOCK $^{17}$ described earlier, we further investigate ZRANK ${ }^{29}$ and DFIRE. ${ }^{30-32}$ ZRANK is an optimized empirical energy function for reranking initial-stage docking predictions. ${ }^{29,33}$ DFIRE is a statistical energy function based on a Distance-scaled, Finite-Ideal-gas REference state. ${ }^{30}$ Originally developed for monomeric proteins, the physical reference state makes the DFIRE energy function directly applicable to protein-protein binding and docking studies. ${ }^{31}$ A method, which integrates ZDOCK for sampling and DFIRE for ranking docking decoys leads to reasonable prediction for four of the six targets (the highest fractions of native contacts in top 10 models for the four targets are $29,54,89$, and $94 \%$, respectively) in the community-wide experiment for assessing blind predictions of protein-protein interactions (CAPRI Rounds 4 and 5). ${ }^{32}$ Combing ZDOCK for sampling and EMPIRE for ranking docking decoys leads to acceptable or medium accuracy in predicting two of the three targets (the highest fractions of native contacts in top 10 models for the two targets are $46 \%$ and 57\%, respectively) in CAPRI rounds 10, 11, and 12 (http://www.ebi.ac.uk/msd-srv/ capri/).

Testing the aforementioned five energy functions indicates that DFIRE is among the best for locating near natives in unrefined structures while EMPIRE is among the best for locating near natives in refined structures. Thus, we propose a DEMPIRE procedure that employs the DFIRE energy function as the initial filter and EMPIRE for final optimization and rescoring. The proposed protocol is tested by using two separated benchmarks. 


\section{METHODS}

\section{Initial near-native decoys}

As in, ref. 18 a near-native structure is a structure with an interface rmsd less than $2.5 \AA$. An interface rmsd between a docking decoy and a native complex structure is based on the $C_{a}$ atoms of interface residues [Interface residues are receptor (or ligand) residues with at least one atom within $10 \AA$ of any atoms of ligand(or receptor)]. We randomly select one nearnative decoy per protein-protein complex from the near-native decoys contained in ZDOCK 2.3 decoy set ${ }^{18}$ (http://zlab.bu.edu/zdock/decoys.shtml). A total of 43 near-native decoy structures are obtained because only 43 of 48 protein-protein complexes in the decoy set contain near-native structures in top 2000 conformations.

\section{Local resampling}

We locally resample the conformational space around the near-native structures obtained above. First, the ligand, the smaller partner of the protein-protein complex, is rotated around a randomly selected axis through the geometry center of the ligand with a step size of a $a=\sqrt{\theta^{2}+\phi^{2}+\psi^{2}}<15^{\circ}$ and $-15^{\circ}<\theta, \varphi \psi<15^{\circ}$, where $\theta, \varphi$, and $\psi$ are three Euler's angles. The random rotation of the ligand is followed by its random translation with a step size of $\Delta r=\sqrt{(\Delta x)^{2}+(\Delta y)^{2}+(\Delta z)^{2}}<2.4 \AA,-2.4 \AA<\Delta x<2.4 \AA,-2.4 \AA<\Delta y<2.4 \AA$, and -2.4 $\AA<\Delta z<2.4 \AA$. This procedure is repeated 2000 times and leads to 2000 new docking decoys per near-native structure. The maximum allowed changes of angles $\left(15^{\circ}\right)$ and displacement $(2.4 \AA)$ are consistent with or doubled from the sampling parameters recommended by ZDOCK $\left(15^{\circ}\right.$ or $6^{\circ}$ in rotation and $1.2 \AA$ in translation $) .{ }^{18}$

\section{Refinement}

The new decoys generated above are subjected to side-chain modeling of the interface residues and further energy minimization. The algorithm of the side-chain modeling and 50 STEP of CHARMM energy minimization ${ }^{34}$ was the same as our previous study. ${ }^{27}$ During side-chain modeling, all backbone structures in a protein-protein complex are fixed.

\section{Scoring functions}

Five energy functions are used to rank the 2000 decoys generated from one near-native structure, after side-chain modeling, and after energy minimization, respectively. They are RDOCK, ZRANK, RosettaDock, DFIRE, and EMPIRE, respectively.

RDOCK $^{17}$ and ZRANK ${ }^{29}$ were developed by Weng's group at Boston University. They were downloaded from http://zlab.bu.edu. RDOCK is a refinement program that refines ZDOCK predictions while ZRANK is a recently developed empirical energy function for reranking protein docking predictions. RosettaDock ${ }^{19}$ is a part of the software code of Rosetta (version 2.1.1) developed by the David Baker's group (http://www.bakerlab.org/). DCOMPLEX and EMPIRE are developed in our group. DCOMPLEX is a program with a built-in DFIRE statistical potential energy function ${ }^{30}$ for protein-protein binding. ${ }^{31}$ EMPIRE is an empirical energy that is designed for selection of near-native conformations among structurally refined docking decoys. ${ }^{27}$

\section{Three stages and two rounds of resampling and refinement}

Side-Chain optimization and energy minimization of all 2000 decoys are time-consuming. For example, it takes 3-12 days to optimize all interface side chains in 2000 decoys on a single 2.2 GHz CPU (Dual Core AMD Opteron Processor 275) dependent on the size of an interface. By comparison, it takes less than 10 min for using DFIRE to evaluate the energy 
values of all these 2000 decoys. Moreover, DFIRE is found among the best for ranking unoptimized decoys and EMPIRE is among the best for ranking optimized one, we further propose a three-stage DEMPIRE refinement procedure: (1) the number of initial (unoptimized) 2000 decoys is reduced to top 15 by DFIRE, (2) the 15 decoys are side-chain optimized and ranked by EMPIRE, ${ }^{27}$ and (3) the top 10 side-chain-optimized decoys are further energy minimized by $\mathrm{CHARMM}^{34}$ (50 ABNR steps ${ }^{27}$ ) and ranked by EMPIRE to produce the final prediction. ${ }^{27}$

We further investigate the effect of a more-refined, second-round local resampling by limiting the maximum allowed angle change to $8^{\circ}$ and maximum translational displacement to $1.2 \AA$. This is performed as follows: We resample the conformational space around the top-1 ranked structure from the first round to generated another 2000 decoys. These 2000 decoys are ranked by DFIRE to produce top 25 decoys. The 25 decoys are side-chain optimized and ranked by EMPIRE ${ }^{27}$ and the top 10 side-chain-optimized decoys are further energy minimized by CHARMM ${ }^{34}$ and ranked by EMPIRE.

\section{Benchmark 2.0}

To further test the proposed method, we also downloaded ZDOCK2.3 docking decoys for Benchmark 2.0 from http://zlab.bu.edu/zdock/decoys.shtml. For the 62 complexes in Benchmark 2.0 excluding antigen-antibody, 42 complexes possess a sequence identity of $50 \%$ or less with any of the 28 targets in enzyme/inhibitor and other protein groups in Benchmark 1.0. Among the 42 complexes, only 15 (1F51 7CEI 1B6C 1BVN 1E6E 1KAC 1EWY 1TMQ 1F34 1AKJ 1ML0 1HE1 1E96 1GRN 1RLB) complexes have near-native decoys in the top 2000 decoys. In addition, there are nine new antigen/antibody complexes (1E6J 1JPS 1BJ1 1FSK 1I9R 1IQD 1K4C 1KXQ 1NSN) in Benchmark 2.0, which have at least one near-native decoy ranked in the top 2000 . These 24 complexes are used as a test set for DEMPIRE.

\section{Assessment}

The accuracy of decoy structures is assessed by interface and global root-mean-squared deviation (irmsd and grmsd, respectively). irmsd is obtained by superposition and RMSD calculation of the $\mathrm{C}_{\mathrm{a}}$ atoms of interface residues, which are receptor (or ligand) residues with at least one atom within $10 \AA$ of any atoms of the ligand (or receptor). grmsd, on the other hand, is obtained by superposing all $\mathrm{C}_{\mathrm{a}}$ atoms of receptor atoms of the docking decoy onto those of the native complex and calculating the rmsd value between ligand atoms of the two complexes.

\section{RESULTS AND DISCUSSION}

Table I compares the ability of five scoring functions for selecting the best near-native structures from 2000 locally resampled (unoptimized), side-chain-optimized, and energyminimized structures. It shows the average interface and global rmsd values of top-1 ranked models selected by five scoring functions for 43 protein-protein complexes. A more detailed table can be found in the supplement material. In Table I, we also define a success rate, the fraction of refined complex structures whose irmsd or grmsd values are reduced by $0.5 \AA$ or more in 43 protein complexes and an error rate, the fraction of refined complex structures whose irmsd or grmsd values are increased by $0.5 \AA$ or more in 43 protein complexes. For locally resampled but unoptimized docking decoys, ZRANK, DFIRE, and EMPIRE yield average irmsd values that are lower than or equal to $1.61 \AA$, the average value for the initial 43 "seed" near-native structures for local sampling. DFIRE has the lowest average irmsd value of $1.49 \AA$, the highest success rate of $23.3 \%$, and a moderate error rate of $11.6 \%$. However, only EMPIRE yields lower interface (or global) rmsd values than $1.61 \AA$ (or 4.88 
$\AA$ ) for side-chain optimized decoys. It is also the only one who has a significantly higher success rate than the error rate ( $>10 \%$ higher). Meanwhile, not a single method improves the predicted models by selecting from further energy minimized decoys (based on either average irmsd values or success/error rates). It should be emphasized that this comparison serves only as an approximate guide because the procedures for side-chain optimization and energy minimization were optimized for EMPIRE and the difference between two methods is not always statistically significant (e.g. $P$-value above 0.1 for DFIRE vs. ZRANK in unoptimized decoys and EMPIRE versus Rosetta in energy-minimized decoys for interface rmsd). Nevertheless, it suggests that DFIRE is among the most suitable for ranking unoptimized structures while EMPIRE is among the most suitable for ranking optimized structures (side-chain optimized, in particular).

The aforementioned result leads to the proposed three-stage scoring and ranking (DFIRE for initial ranking and enrichment, EMPIRE for ranking side-chain optimized and energy minimized decoys). This three-stage refinement protocol is performed for two separate rounds and results are shown in Table II. The average interface rmsd value reduces from 1.61 to $1.36 \AA$ in the first round and to $1.25 \AA$ in the second round while the average global rmsd value reduces from 4.88 to $3.87 \AA$ in the first round and $3.54 \AA$ in the second round. The final mean irmsd $(1.25 \AA)$ and grmsd $(3.54 \AA)$ results are significantly lower than the corresponding values from random selections as a control (2.18 and $6.04 \AA$, respectively). The differences between the final and initial rmsd values and between the final and randomselection rmsd values according to paired $T$-test (GraphPad Software: http:// www.graphpad.com/quickcalcs/ttest1.cfm) are all statistically significant with $P$-value < 0.0001 . The most encouraging result is that only a few predicted complexes by the proposed protocol have an increase of rmsd (global or interface) for more than $0.5 \AA$, whereas the majority (74\% after the 2 nd round) has a large reduction of global rmsd values (1.9 $\AA$ in average).

Figure 1 illustrates two successful examples (1SPB and 1UDI). The native complex structure is colored in red. The improvement from the initial structure (in green) is clearly visible after locally refinements with DEMPIRE (the first round in blue and the second round in orange).

In both rounds of resampling, we selected top 10 side-chain-optimized decoys for energy minimization. The numbers of decoys selected for side-chain modeling (15 in the first round and 25 in the second round) were chosen by trial and error. In the first round resampling, when $10,15,20,25$, and 30 decoys were selected for side-chain modeling, the mean values of interface rmsd were $1.37,1.36,1.38,1.43$, and $1.41 \AA$, respectively, for the top ranked decoy of the 43 targets. In the second round resampling, when 10, 15, 20, 25, 30, 35, 40, 100 , and 200 decoys were selected for side-chain modeling, the mean values of the interface rmsd were $1.33,1.32,1.27,1.25,1.28,1.28,1.28,1.31$, and $1.31 \AA$, respectively. Here, the step size of the grid search was set to 5 . We have also tried selecting 5 or 15 decoys for energy minimization, but the prediction accuracy was not improved even if we changed the number of decoys selected for side chain modeling. These parameters optimized with enzyme/inhibitor and other proteins group were also the most effective ones for the antigen/ antibody group though the two groups of targets have a quite different interface properties. This reflects the transferability of the DFIRE ${ }^{31}$ and EMPIRE $^{27}$ energy functions due to their inherent physical origins.

The two-round refinement protocol is further tested on 24 complexes in Benchmark 2.0. The results are shown in Table III. The improvement is not as significant as 43 complexes in Benchmark 1.0 (Table II). The average irmsd and grmsd decrease by 0.14 and $0.33 \AA$, respectively. There are only four complexes (17\%) with greater than $0.5 \AA$ improvement in 
interface rmsd values and nine (38\%) with greater than $0.5 \AA$ improvement in global rmsd values after the second round of sampling and refinement while the predicted models of two or $8 \%$ (eight, or $33 \%$ ) complexes have increased interface (global) rmsd values for more than $0.5 \AA$. Moreover, the difference between the structural accuracy of the final result (after the second round) and that of the initial decoy is only marginal ( $P$-value $=0.1$ for irmsd) or not statistical significant $(P$-value $=0.3$ for grmsd $)$. Nevertheless, improvement over random prediction is significant $(P$-value $<0.0001$ for irmsd and grmsd). The number of complexes with improved prediction accuracy is consistently larger than the number of complexes with worse prediction accuracy (in either irmsd or grmsd values).

To further understand the reason behind the difference between refinement result for protein complexes in Benchmark 1.0 and that in Benchmark 2.0, we compare irmsd values of the initial structures with those of final predicted structures in Figure 2. There is an obvious difference between the initial near-native structures in Benchmark 1.0 and those in Benchmark 2.0. The former have significantly lower irmsd values in average than the latter. In fact, all initial near-native structures in Benchmark 2.0 have an irmsd value greater than $1.27 \AA$. By comparison, there are 13 initial near-native structures in Benchmark 1.0 with irmsd $<1.27 \AA$. In addition, the average number of near-native structures predicted by ZDOCK 2.3 for Benchmark 2.0 (34 for 24 complexes) in top 2000 decoys is significantly smaller than that for Benchmark 1.0 (51 for 43 complexes). This suggests that Benchmark 2.0 is inherently much more difficult than Benchmark 1.0 for ZDOCK2.3 as well.

Interestingly, the sharp difference in the performance is also observed when a method called PINUP (Protein Interface residUe Prediction) is employed to predict binding regions for the protein complexes in the two benchmarks. ${ }^{35}$ It was found that binding residues of protein complexes in Benchmark 2.0 is less conserved than those residues of protein complexes in Benchmark 1.0. ${ }^{35}$ Thus, the observed difference may simply reflect the different interface properties between Benchmarks 1.0 and 2.0.

\section{CONCLUDING REMARKS}

In this article, we showed that there is a lack of a scoring function to locate a model from many sampled structures whose structure is more accurate than the starting near-native structure (Table I). Thus, we propose to use the DFIRE energy to filter locally sampled but unoptimized structures. Only those filtered structures are subjected to side-chain optimization, energy minimization, and ranking by EMPIRE. We demonstrated that this protocol (DEMPIRE) is not only computational efficient but more importantly improve predicted models. Significant improvement (74\% complex structures improved over $0.5 \AA$ or more than starting near-native structures in global rmsd values) is observed for 43 targets in Benchmark 1.0 while less significant result (38\% complex structures improved over $0.5 \AA$ or more than starting near-native structures in global rmsd values) is obtained for 24 targets in Benchmark 2.0.

\section{Supplementary Material}

Refer to Web version on PubMed Central for supplementary material.

\section{Acknowledgments}

Grant sponsor: NIH; Grant numbers: GM066049, GM085003; Grant sponsor: Natural Science Foundation of China; Grant number: 30400096. 


\section{REFERENCES}

1. Valencia A. Protein refinement: A new challenge for CASP in its 10th anniversary. Bioinformatics. 2005; 21:277-277. [PubMed: 15647301]

2. Misura KM, Baker D. Progress and challenges in high-resolution refinement of protein structure models. Proteins. 2005; 59:15-29. [PubMed: 15690346]

3. Zhang Y, Skolnick J. Automated structure prediction of weakly homologous proteins on a genomic scale. Proc Natl Acad Sci USA. 2004; 101:7594-7599. [PubMed: 15126668]

4. Marti-Renom M, Stuart A, Fiser A, Sanchez R, Melo F, Šali A. Comparative protein structure modeling of genes and genomes. Annu Rev Biophys Biomol Struct. 2000; 29:291-325. [PubMed: 10940251]

5. Lu H, Skolnick J. Application of statistical potentials to protein structure refinement from low resolution ab initio models. Biopolymers. 2003; 70:575-584. [PubMed: 14648767]

6. Fan H, Mark AE. Refinement of homology-based protein structures by molecular dynamics simulation techniques. Protein Sci. 2004; 13:211-220. [PubMed: 14691236]

7. Wallner B, Elofsson A. All are not equal: a benchmark of different homology modeling programs. Protein Sci. 2005; 14:1315-1327. [PubMed: 15840834]

8. Misura KMS, Chivian D, Rohl CA, Kim DE, Baker D. Physically realistic homology models built with ROSETTA can be more accurate than their templates. Proc Natl Acad Sci USA. 2006; 103:5361-5366. [PubMed: 16567638]

9. Zhu J, Xie L, Honig B. Structural refinement of protein segments containing secondary structure elements: Local sampling, knowledge-based potentials and clustering. Proteins. 2006; 65:463-479. [PubMed: 16927337]

10. Summa CM, Levitt M. Near-native structure refinement using in vacuo energy minimization. Proc Natl Acad Sci USA. 2007; 104:3177-3182. [PubMed: 17360625]

11. Chen J, Charles L, Brooks I. Can molecular dynamics simulations provide high-resolution refinement of protein structure? Proteins. 2007; 67:922-930. [PubMed: 17373704]

12. Jackson RM, Sternberg MJE. A continuum model for protein-protein interactions: application to the docking problem. J Mol Biol. 1995; 250:258-275. [PubMed: 7541840]

13. Weng Z, Vajda S, Delisi C. Prediction of protein complexes using empirical free energy functions. Protein Sci. 1996; 5:614-626. [PubMed: 8845751]

14. Smith GR, Sternberg MJE. Prediction of protein-protein interactions by docking methods. Curr Opin Struct Biol. 2002; 12:28-35. [PubMed: 11839486]

15. Lorber DM, Udo MK, Shoichet BK. Protein-protein docking with multiple residue conformations and residue substitutions. Protein Sci. 2002; 11:1393-1408. [PubMed: 12021438]

16. Fernandez-Recio J, Totrov M, Abagyan R. Soft protein-protein docking in internal coordinates. Protein Sci. 2002; 11:280-291. [PubMed: 11790838]

17. Li L, Chen R, Weng Z. RDOCK: refinement of rigid-body protein docking predictions. Proteins. 2003; 53:693-707. [PubMed: 14579360]

18. Chen R, Li L, Weng Z. ZDOCK: an initial-stage protein-docking algorithm. Proteins. 2003; 52:8087. [PubMed: 12784371]

19. Gray J, Moughan S, Wang C, Schueler-Furman O, Kuhlman B, Rohl C, Baker D. Protein-protein docking with simultaneous optimization of rigid body displacement and side chain conformations. J Mol Biol. 2003; 331:281-299. [PubMed: 12875852]

20. Daily MD, Masica D, Sivasubramanian A, Somarouthu S, Gray JJ. CAPRI rounds 3-5 reveal promising successes and future challenges for RosettaDock. Proteins. 2005; 60:181-186.

[PubMed: 15981262]

21. Schueler-Furman O, Wang C, Baker D. Progress in protein-protein docking: atomic resolution predictions in the CAPRI experiment using RosettaDock with an improved treatment of side-chain flexibility. Proteins. 2005; 60:187-194. [PubMed: 15981249]

22. Chaudhury S, Sircar A, Sivasubramanian A, Berrondo M, Gray JJ. Incorporating biochemical information and backbone flexibility in RosettaDock for CAPRI rounds 6-12. Proteins. 2007; 69:793-800. [PubMed: 17894347] 
23. Wang C, Schueler-Furman O, Andre I, London N, Fleishman SJ, Bradley P, Qian B, Baker D. RosettaDock in CAPRI rounds 6-12. Proteins. 2007; 69:758-763. [PubMed: 17671979]

24. Carter P, Lesk VI, Islam SA, Sternberg MJ. Protein-protein docking using 3D-Dock in rounds 3, 4, and 5 of CAPRI. Proteins. 2005; 60:281-288. [PubMed: 15981271]

25. Jackson RM, Gabb HA, Sternberg MJ. Rapid refinement of protein interfaces incorporating solvation: application to the docking problem. J Mol Biol. 1998; 276:265-285. [PubMed: 9514726]

26. Kowalsman N, Eisenstein M. Inherent limitations in protein-protein docking procedures. Bioinformatics. 2007; 23:421-426. [PubMed: 17040920]

27. Liang S, Liu S, Zhang C, Zhou Y. A simple reference state makes a significant improvement in near-native selections from structurally refined docking decoys. Proteins. 2007; 69:244-253. [PubMed: 17623864]

28. Camacho CJ, Vajda S. Protein docking along smooth association pathways. Proc Natl Acad Sci USA. 2001; 98:10636-10641. [PubMed: 11517309]

29. Pierce B, Weng Z. ZRANK: reranking protein docking predictions with an optimized energy function. Proteins. 2007; 67:1078-1086. [PubMed: 17373710]

30. Zhou H, Zhou Y. Distance-scaled, finite ideal-gas reference state improves structure-derived potentials of mean force for structure selection and stability prediction. Protein Sci. 2002; 11:2714-2726. [PubMed: 12381853]

31. Liu S, Zhang C, Zhou H, Zhou Y. A physical reference state unifies the structure-derived potential of mean force for protein folding and binding. Proteins. 2004; 56:93-101. [PubMed: 15162489]

32. Zhang C, Liu S, Zhou Y. Docking prediction using biological information, ZDOCK sampling technique and clusterization guided by the DFIRE statistical energy function. Proteins. 2005; 60:314-318. [PubMed: 15981255]

33. Wiehe K, Pierce B, Tong WW, Hwang H, Mintseris J, Weng Z. The performance of ZDOCK and ZRANK in rounds 6-11 of CAPRI. Proteins. 2007; 69:719-725. [PubMed: 17803212]

34. Brooks BR, Bruccoleri RE, Olafson BD, States DJ, Swaminathan S, Karplus M. CHARMM: a program for macromolecular energy, minimization, and dynamics calculations. J Comput Chem. $1983 ; 4: 187-217$.

35. Liang S, Zhang C, Liu S, Zhou Y. Protein binding site prediction with an empirical scoring function. Nucleic Acids Res. 2006; 34:3698-3707. [PubMed: 16893954] 
(a)

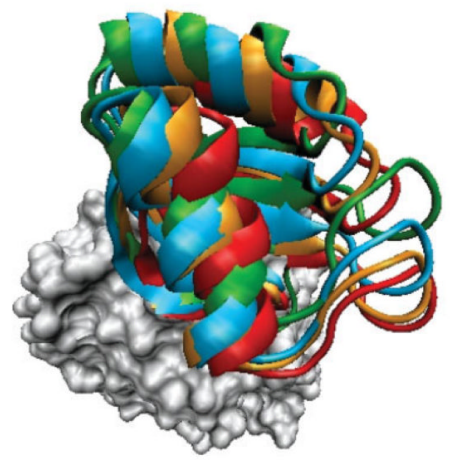

(b)

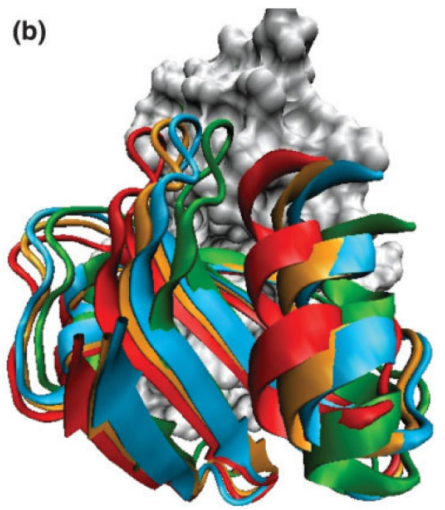

Figure 1.

Two successful examples illustrate the structural change from the initial near-native structure (in green) to the local refinement after the first round (blue) and after the second round (orange). The native conformation is shown in red. (a) 1spb. (b) 1udi. 


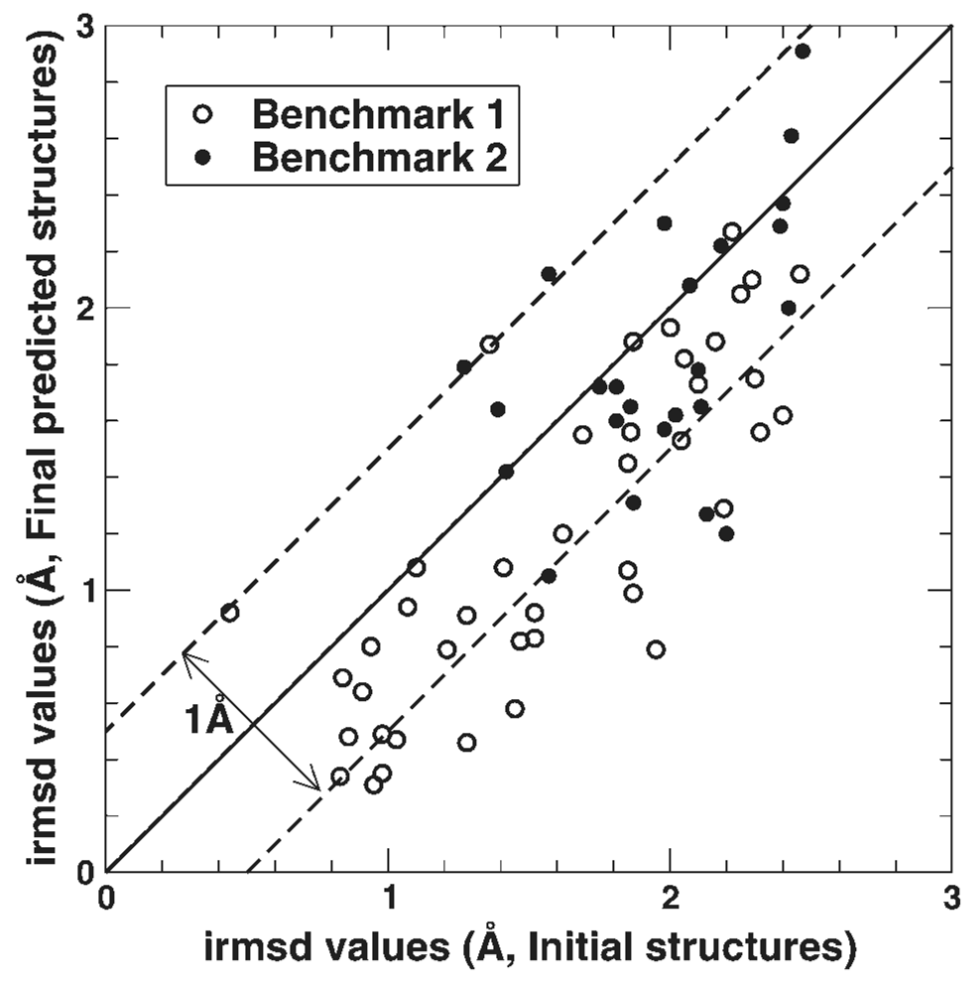

Figure 2.

The interface rmsd values of the initial near-native structures versus those of the final predicted structures in Benchmarks 1.0 (open circles) and 2.0 (filled circles). Solid line separates the regions where refinement leads to improved structures (lower triangle) and where less-accurate structures are obtained (upper triangle). Dashed lines indicate the region where the absolute difference between the irmsd value of the initial near-native structure and that of the final structure is less than $0.5 \AA$. 


\section{Table I}

The Average Interface and Global rmsd Values of the Top 1-Ranked Models of 43 Protein-Protein Complexes Selected by Five Different Scoring Functions From Locally Resampled Structures Around a Randomly Selected Near-Native Structure Per Complex

\begin{tabular}{|c|c|c|c|c|c|c|}
\hline & \multicolumn{3}{|c|}{ Interface rmsd ( $(\AA){ }^{a}$ (Succ. rate, ${ }^{b}$ Error rate ${ }^{c}$ ) } & \multicolumn{3}{|c|}{ Global rmsd (£) ${ }^{d}$ (Succ. rate, ${ }^{b}$ Error rate $\left.{ }^{c}\right)$} \\
\hline & Local $^{e}$ & Side-chain $f$ & Energy $g$ & Local $^{e}$ & Side-chain $f$ & Energy $g$ \\
\hline RosettaDock $^{h}$ & $1.80(4.7 \%, 25.6 \%)$ & $1.86(7.0 \%, 23.3 \%)$ & $1.75(9.3 \%, 20.9 \%)$ & $4.98(25.6 \%, 34.9 \%)$ & $5.37(25.6 \%, 48.8 \%)$ & $5.03(30.2 \%, 32.6 \%)$ \\
\hline $\mathrm{RDOCK}^{i}$ & - & - & $1.85(7.0 \%, 30.2 \%)$ & - & - & $5.27(30.2 \%, 48.8 \%)$ \\
\hline $\mathrm{ZRANK}^{j}$ & $1.54(9.3 \%, 7.0 \%)$ & $1.97(7.0 \%, 39.5 \%)$ & $2.53(0.0 \%, 74.4 \%)$ & $4.47(46.5 \%, 25.6 \%)$ & $5.98(18.6 \%, 67.4 \%)$ & $6.35(7.0 \%, 81.4 \%)$ \\
\hline DFIRE $^{j}$ & $1.49(23.3 \%, 11.6 \%)$ & $1.60(20.9 \%, 16.3 \%)$ & $2.10(0 \%, 48.8 \%)$ & $4.27(53.5 \%, 27.9 \%)$ & $4.80(39.5 \%, 39.5 \%)$ & $5.74(16.3 \%, 67.4 \%)$ \\
\hline EMPIRE $^{j}$ & $1.61(20.9 \%, 16.3 \%)$ & $1.52(25.6 \%, 14.0 \%)$ & $1.61(11.6 \%, 11.6 \%)$ & $4.5(44.2 \%, 23.3 \%)$ & $4.47(55.8 \%, 23.3 \%)$ & $5.00(34.9 \%, 39.5 \%)$ \\
\hline
\end{tabular}

The average interface and global rmsd values for initial 43 near-native structures are 1.61 and $4.88 \AA$, respectively. The success (or error) rates for decreasing (or increasing) irmsd or grmsd values by more than $0.5 \AA$ in 43 targets are also shown.

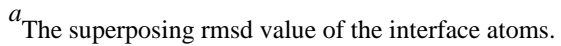

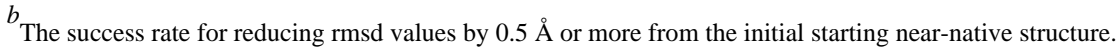

$c_{\text {The error rate for increasing rmsd values by } 0.5 \AA \text { or more. }}$

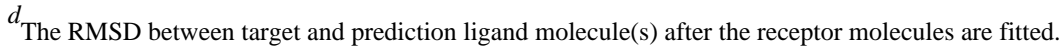

$e_{\text {The initial }} 2000$ decoys generated by random rotation and translation around a near-native structure.

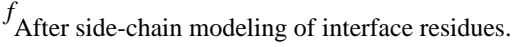

${ }^{g}$ After further energy minimization with CHARMM for 50 ABNR steps.

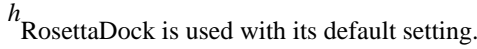

${ }^{i}$ Three stages of energy minimization(removing clashes, optimizing polar interactions, and optimizing of charge interactions) are separately performed according to RDOCK protocol. RDOCK is only applied for energy minimized structures without side-chain modeling.

$j_{\text {ZRANK, DFIRE, and EMPIRE energy functions are directly applied to the decoys. }}$ 
Table II

The Average Interface and Global rmsd Values of the Top-1 Ranked Models of 43 Protein-Protein Complexes by Two Rounds of Local Resampling Plus Three Stages of Scoring and Refinements by DFIRE and EMPIRE

\begin{tabular}{|c|c|c|c|c|c|c|c|c|}
\hline \multirow[b]{2}{*}{ PDB } & \multicolumn{4}{|c|}{ Interface rmsd $(\AA)^{a}$} & \multicolumn{4}{|c|}{ Global rmsd $(\AA)^{b}$} \\
\hline & Initial $c$ & 1st Round $d$ & 2nd Round ${ }^{e}$ & Random $f$ & Initial $c$ & 1st Round $d$ & 2nd Round ${ }^{e}$ & Random $f$ \\
\hline 1CGI & 2.46 & 2.36 & 2.12 & 3.00 & 5.44 & 5.62 & 5.13 & 6.31 \\
\hline $1 \mathrm{CHO}$ & 1.10 & 1.13 & 1.08 & 1.26 & 3.66 & 4.25 & 4.11 & 3.65 \\
\hline 2PTC & 0.44 & 0.86 & 0.92 & 1.66 & 1.26 & 2.65 & 3.17 & 3.79 \\
\hline 1TGS & 1.69 & 1.62 & 1.55 & 2.69 & 3.15 & 2.49 & 1.99 & 5.02 \\
\hline 2SNI & 2.22 & 2.38 & 2.27 & 2.55 & 8.06 & 8.14 & 7.47 & 8.16 \\
\hline 2SIC & 2.32 & 1.77 & 1.56 & 2.65 & 11.60 & 9.36 & 8.63 & 14.10 \\
\hline 1CSE & 2.19 & 1.46 & 1.29 & 2.84 & 7.01 & 4.96 & 4.31 & 8.16 \\
\hline 2KAI & 2.30 & 1.80 & 1.75 & 2.13 & 9.22 & 7.11 & 6.98 & 8.93 \\
\hline 1BRC & 1.36 & 1.80 & 1.87 & 2.05 & 6.09 & 7.55 & 7.66 & 8.27 \\
\hline $1 \mathrm{ACB}$ & 1.52 & 1.02 & 0.83 & 2.20 & 4.21 & 2.58 & 1.95 & 5.40 \\
\hline 1BRS & 2.04 & 1.40 & 1.53 & 2.80 & 4.64 & 3.12 & 3.23 & 5.47 \\
\hline $1 \mathrm{MAH}$ & 0.84 & 0.69 & 0.69 & 1.33 & 1.55 & 1.05 & 1.41 & 2.96 \\
\hline $1 \mathrm{UGH}$ & 1.85 & 1.68 & 1.45 & 1.77 & 4.51 & 3.73 & 3.26 & 3.85 \\
\hline 1DFJ & 1.62 & 1.16 & 1.20 & 3.23 & 2.81 & 1.36 & 2.08 & 5.07 \\
\hline 1FSS & 1.07 & 0.95 & 0.94 & 1.29 & 2.36 & 1.93 & 1.64 & 3.02 \\
\hline $1 \mathrm{AVW}$ & 2.00 & 2.01 & 1.93 & 2.97 & 8.88 & 8.88 & 9.05 & 10.60 \\
\hline 1PPE & 1.52 & 1.22 & 0.92 & 2.00 & 4.31 & 3.39 & 2.38 & 5.53 \\
\hline $1 \mathrm{TAB}$ & 1.21 & 0.68 & 0.79 & 1.38 & 3.54 & 1.96 & 2.36 & 3.44 \\
\hline 1UDI & 1.95 & 1.10 & 0.79 & 3.11 & 4.31 & 2.42 & 1.61 & 5.41 \\
\hline $1 \mathrm{STF}$ & 0.83 & 0.60 & 0.34 & 1.52 & 3.01 & 1.70 & 1.11 & 3.90 \\
\hline 2TEC & 0.98 & 0.45 & 0.35 & 1.80 & 2.82 & 1.42 & 0.92 & 4.25 \\
\hline 4HTC & 1.28 & 0.81 & 0.91 & 2.32 & 3.09 & 1.38 & 1.44 & 4.55 \\
\hline 1WQ1 & 2.29 & 2.02 & 2.10 & 3.59 & 7.87 & 6.14 & 5.98 & 9.39 \\
\hline 1IGC & 1.47 & 0.76 & 0.82 & 1.33 & 3.42 & 1.32 & 1.55 & 2.58 \\
\hline $1 \mathrm{ATN}$ & 0.95 & 0.52 & 0.31 & 1.99 & 3.81 & 1.18 & 0.48 & 5.46 \\
\hline 1SPB & 1.45 & 0.91 & 0.58 & 2.81 & 4.65 & 3.06 & 1.78 & 6.50 \\
\hline $2 \mathrm{BTF}$ & 1.28 & 0.61 & 0.46 & 2.58 & 2.35 & 1.62 & 1.31 & 4.34 \\
\hline $1 \mathrm{~A} 0 \mathrm{O}$ & 2.25 & 2.25 & 2.05 & 2.27 & 4.05 & 5.37 & 4.22 & 4.67 \\
\hline $1 \mathrm{MLC}$ & 2.10 & 2.17 & 1.73 & 2.55 & 6.36 & 5.68 & 4.52 & 7.50 \\
\hline 1WEJ & 0.91 & 0.75 & 0.64 & 1.56 & 2.12 & 1.55 & 1.21 & 4.40 \\
\hline 1AHW & 1.87 & 1.52 & 1.88 & 1.78 & 7.00 & 5.80 & 6.64 & 3.90 \\
\hline $1 \mathrm{BVK}$ & 2.40 & 3.62 & 3.70 & 2.27 & 6.09 & 6.96 & 7.25 & 4.36 \\
\hline 1FBI & 2.05 & 2.33 & 1.82 & 1.69 & 4.09 & 4.09 & 3.32 & 3.83 \\
\hline 2JEL & 2.16 & 1.65 & 1.88 & 2.42 & 6.97 & 5.68 & 6.72 & 8.20 \\
\hline $1 \mathrm{BQL}$ & 1.86 & 2.04 & 1.56 & 2.79 & 5.03 & 5.19 & 4.09 & 6.53 \\
\hline 1JHL & 1.85 & 1.31 & 1.07 & 2.35 & 4.97 & 4.15 & 3.73 & 4.77 \\
\hline $1 \mathrm{NCA}$ & 0.86 & 0.89 & 0.48 & 1.19 & 3.74 & 2.84 & 0.99 & 2.74 \\
\hline
\end{tabular}




\begin{tabular}{|c|c|c|c|c|c|c|c|c|}
\hline \multirow[b]{2}{*}{ PDB } & \multicolumn{4}{|c|}{ Interface rmsd $(\AA)^{a}$} & \multicolumn{4}{|c|}{ Global rmsd $(\AA)^{b}$} \\
\hline & Initial $^{c}$ & 1st Round ${ }^{d}$ & 2nd Round ${ }^{e}$ & Random $f$ & Initial $^{c}$ & 1st Round ${ }^{d}$ & 2nd Round ${ }^{e}$ & Random $f$ \\
\hline $1 \mathrm{NMB}$ & 0.98 & 0.59 & 0.49 & 3.22 & 3.27 & 2.80 & 1.06 & 14.10 \\
\hline 1MEL & 1.41 & 1.17 & 1.08 & 2.64 & 5.11 & 2.13 & 1.89 & 12.60 \\
\hline 2VIR & 1.03 & 0.91 & 0.47 & 1.22 & 2.80 & 3.50 & 1.71 & 3.66 \\
\hline $1 \mathrm{EO} 8$ & 0.94 & 1.03 & 0.80 & 2.82 & 4.11 & 3.35 & 3.74 & 10.90 \\
\hline $1 \mathrm{QFU}$ & 1.87 & 0.92 & 0.99 & 3.03 & 7.21 & 2.72 & 2.87 & 9.25 \\
\hline 1IAI & 2.40 & 1.45 & 1.62 & 2.86 & 9.18 & 4.43 & 5.18 & 10.00 \\
\hline Mean $g$ & 1.61 & 1.36 & 1.25 & 2.27 & 4.88 & 3.87 & 3.54 & 6.27 \\
\hline \multicolumn{2}{|c|}{ Succ. rate ${ }^{h}$} & $15 / 43(35 \%)$ & $16 / 43(37 \%)$ & $0 / 43(0 \%)$ & & $30 / 43(70 \%)$ & $32 / 43(74 \%)$ & $5 / 43(12 \%)$ \\
\hline \multicolumn{2}{|c|}{ Error rate $e^{i}$} & $1 / 43(2 \%)$ & $2 / 43(5 \%)$ & $24 / 43(56 \%)$ & & $6 / 43(14 \%)$ & $3 / 43(7 \%)$ & $32 / 43(74 \%)$ \\
\hline
\end{tabular}

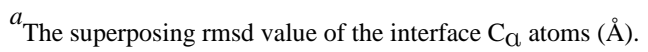

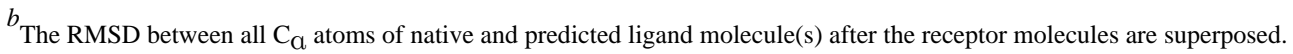

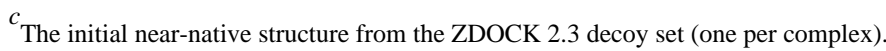

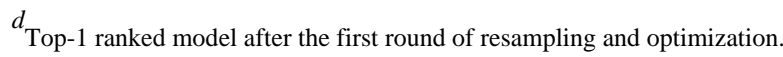

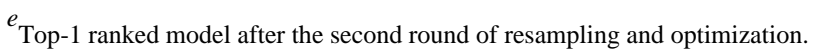

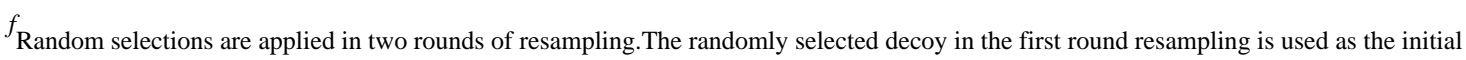
structure for the second round resampling.

$g_{\text {The average value of } 43 \text { complex structures. }}$

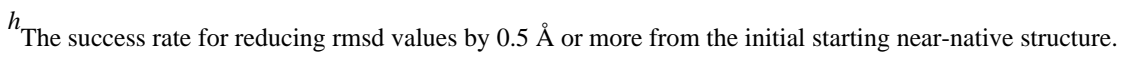

${ }^{i}$ The error rate for increasing rmsd values by $0.5 \AA$ or more. 


\section{Table III}

The Average Interface and Global rmsd Values of the top-1 Ranked Models of 24 Protein-Protein Complexes by Local Resampling Plus Three Stages of Scoring and Refinements by DFIRE and EMPIRE (Benchmark 2.0)

\begin{tabular}{|c|c|c|c|c|c|c|c|c|}
\hline \multirow[b]{2}{*}{ PDB } & \multicolumn{4}{|c|}{ Interface rmsd $(\AA)^{a}$} & \multicolumn{4}{|c|}{ Global rmsd $(\AA) b$} \\
\hline & Initial $^{c}$ & 1st Round $d$ & 2nd Round $e$ & Random $f$ & Initial $^{c}$ & 1st Round $d$ & 2nd Round ${ }^{e}$ & Random $f$ \\
\hline 1F51 & 1.27 & 1.96 & 1.79 & 2.41 & 1.67 & 3.12 & 2.84 & 4.62 \\
\hline 7CEI & 2.11 & 1.77 & 1.65 & 3.26 & 11.5 & 8.41 & 6.17 & 16.60 \\
\hline 1B6C & 2.18 & 2.27 & 2.22 & 2.68 & 4.91 & 4.43 & 4.11 & 10.10 \\
\hline $1 \mathrm{BVN}$ & 1.57 & 1.63 & 2.12 & 2.10 & 3.52 & 3.64 & 4.50 & 5.61 \\
\hline 1E6E & 2.10 & 1.54 & 1.78 & 3.28 & 4.36 & 2.99 & 2.94 & 6.57 \\
\hline $1 \mathrm{KAC}$ & 2.42 & 2.07 & 2.00 & 3.12 & 7.78 & 7.05 & 6.93 & 9.23 \\
\hline 1EWY & 1.87 & 1.81 & 1.31 & 3.05 & 3.67 & 3.53 & 2.45 & 6.16 \\
\hline 1TMQ & 1.81 & 1.75 & 1.60 & 2.59 & 5.23 & 6.28 & 5.29 & 6.83 \\
\hline 1F34 & 1.98 & 1.90 & 1.57 & 3.52 & 3.91 & 4.81 & 4.27 & 6.94 \\
\hline $1 \mathrm{AKJ}$ & 2.02 & 1.54 & 1.62 & 4.39 & 5.11 & 4.07 & 4.52 & 8.84 \\
\hline 1ML0 & 2.20 & 1.37 & 1.20 & 1.86 & 5.42 & 3.20 & 2.30 & 4.31 \\
\hline 1HE1 & 1.75 & 1.84 & 1.72 & 2.64 & 4.52 & 4.86 & 5.04 & 8.31 \\
\hline 1E96 & 2.39 & 2.40 & 2.29 & 2.84 & 8.63 & 10.30 & 9.82 & 11.80 \\
\hline $1 \mathrm{GRN}$ & 2.47 & 2.85 & 2.91 & 2.91 & 5.92 & 7.63 & 7.32 & 8.66 \\
\hline 1RLB & 2.43 & 2.66 & 2.61 & 2.66 & 13.6 & 15.20 & 14.40 & 11.80 \\
\hline $1 \mathrm{E} 6 \mathrm{~J}$ & 1.86 & 1.61 & 1.65 & 2.59 & 4.88 & 5.04 & 5.29 & 4.87 \\
\hline $1 \mathrm{JPS}$ & 2.40 & 2.47 & 2.37 & 2.56 & 9.01 & 9.46 & 8.87 & 10.60 \\
\hline 1BJ1 & 2.07 & 2.36 & 2.08 & 2.47 & 14.7 & 14.90 & 14.80 & 15.60 \\
\hline 1FSK & 1.57 & 1.36 & 1.05 & 2.11 & 4.53 & 3.60 & 2.80 & 6.73 \\
\hline $1 \mathrm{I} 9 \mathrm{R}$ & 1.98 & 2.21 & 2.30 & 4.08 & 11.6 & 12.50 & 12.40 & 15.40 \\
\hline 1IQD & 2.13 & 1.42 & 1.27 & 4.15 & 7.48 & 7.28 & 6.22 & 8.03 \\
\hline $1 \mathrm{~K} 4 \mathrm{C}$ & 1.42 & 1.23 & 1.42 & 1.47 & 5.91 & 5.83 & 5.94 & 4.06 \\
\hline $1 \mathrm{KXQ}$ & 1.39 & 1.37 & 1.64 & 2.99 & 4.48 & 4.48 & 5.68 & 6.47 \\
\hline $1 \mathrm{NSN}$ & 1.81 & 2.22 & 1.72 & 2.94 & 5.14 & 5.32 & 4.70 & 6.75 \\
\hline Mean & 1.97 & 1.90 & 1.83 & 2.86 & 6.56 & 6.58 & 6.23 & 8.54 \\
\hline \multicolumn{2}{|c|}{ Succ. rate $g$} & $3 / 24(12.5 \%)$ & $4 / 24(16.7 \%)$ & $0 / 24(0 \%)$ & & $6 / 24(25.0 \%)$ & $9 / 24(37.5 \%)$ & $3 / 24(13 \%)$ \\
\hline \multicolumn{2}{|c|}{ Error rate $^{h}$} & $1 / 24(4.2 \%)$ & $2 / 24(8.3 \%)$ & $18 / 24(75 \%)$ & & $7 / 24(29.2 \%)$ & $8 / 24(33.3 \%)$ & $20 / 24(83 \%)$ \\
\hline
\end{tabular}

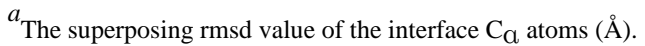

${ }^{b}$ The RMSD between all $\mathrm{C}_{\mathrm{Q}}$ atoms of native and predicted ligand molecule(s) after the receptor molecules are superposed.

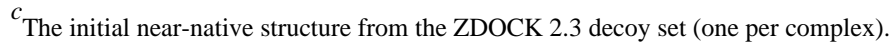

${ }^{d}$ Top-1 ranked model after the first round of resampling and optimization.

$e_{\text {Top-1 }}$ ranked model after the second round of resampling and optimization.

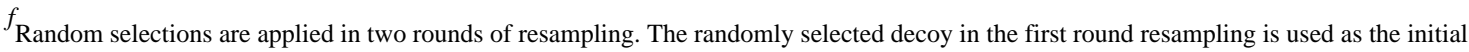
structure for the second round resampling. 
$g_{\text {The average value of } 43 \text { complex structures. }}$

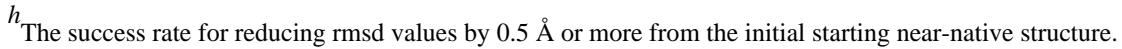

${ }^{i}$ The error rate for increasing rmsd values by $0.5 \AA$ or more. 